\title{
Towards Mobile/Wearable Device Electrosmog Reduction through Careful Network Selection
}

\author{
Jean-Marc Seigneur \\ University of Geneva \\ 7 , route de Drize \\ Carouge, Switzerland \\ Jean-Marc.Seigneur@unige.ch
}

\author{
Xavier Titi \\ University of Geneva \\ 7 , route de Drize \\ Carouge, Switzerland \\ Xavier.Titi@unige.ch
}

\author{
Tewfiq El Maliki \\ HES-SO Geneva \\ 4, rue de la Prairie \\ Genève, Switzerland \\ Tewfiq.EImaliki@hesge.ch
}

\begin{abstract}
There is some concern regarding the effect of smart phones and other wearable devices using wireless communication and worn by the users very closely to their body. In this paper, we propose a new network switching selection model and its algorithms that minimize the non-ionizing radiation of these devices during use. We validate the model and its algorithms with a proof-of-concept implementation on the Android platform.
\end{abstract}

\section{Categories and Subject Descriptors}

C.1.2 [Network Architecture and Design]: Wireless Communication. H.1.2 [User/Machine Systems]: Human Factors. K.4.1 [Public Policy Issues]: Human Safety. K.6.2 [Installation Management]: Performance and usage measurement.

\section{General Terms}

Algorithms, Management, Measurement, Performance, Human Factors

\section{Keywords}

electrosmog, wireless hand-over.

\section{INTRODUCTION}

There are more and more wireless products that are carried out by users from broadly used mobile phones to more specific devices such as cardio belts and watches to monitor heart rates whilst practicing sport. These devices use different wireless technologies to communicate between each other and their Internet remote servers, for example, to store the sport session data. Those devices bring interesting aspects for the users. However, there is some raising concern about the effect of the non-ionizing electromagnetic radiations of the wireless devices on the user's health. Those electromagnetic radiation exposures are generally coined "electrosmog". Non-ionizing radiations mean that they do

Permission to make digital or hard copies of all or part of this work for personal or classroom use is granted without fee provided that copies are not made or distributed for profit or commercial advantage and that copies bear this notice and the full citation on the first page. To copy otherwise, or republish, to post on servers or to redistribute to lists, requires prior specific permission and/or a fee.

Augmented Human Conference, April 2-3, 2010, Megève, France.

Copyright 2010 ACM 978-1-60558-825-4/10/04 ..\$10.00. not carry enough energy per quantum to remove an electron from an atom or molecule.

Section 2 presents the related work. Section 3 surveys the main different wireless technologies used by mobile devices from the point of view of their electromagnetic radiated emission. In Section 4, we propose our networks switching selection model and algorithms to minimize exposures to electrosmog and we validate them via discussing a proof-of-concept implementation. Section 5 concludes the paper.

\section{RELATED WORK}

The potential harmful effects of electrosmog have been researched in many occasions and there are still doubts regarding these effects beyond the transformation of electromagnetic energy in thermal energy in tissues. However, even a sceptical recent survey [1] underlines that the precautionary principle, meaning that efforts for minimizing exposure, should be followed, especially for teenagers.

One of the first means to reduce exposure, besides stopping using it or using it only when needed and in good conditions (close to the base station...), is to use a mobile phone with low Specific Absorption Rate (SAR). However, as the SAR indicated on the mobile phones is measured at their full power strength, some phones with higher SAR may better manage their power strength and end up emitting less than phones with lower SAR that emit more often at full power even if it is not needed. In the USA, the FCC has set a SAR limit of $1.6 \mathrm{~W} / \mathrm{kg}$, averaged over a volume of 1 gram of tissue in the head and in any 6 minute period. In Europe, the ICNIRP limit is $2 \mathrm{~W} / \mathrm{kg}$, averaged over a volume of 10 grams of tissue in the head and in any 6 minute period. Interestingly, the iPhone user manual underlines that it may give a higher SAR than the regulation if used in direct contact with the body: "for body-worn operation, iPhone's SAR measurement may exceed the FCC exposure guidelines if positioned less than 15 $\mathrm{mm}$ (5/8 inch) from the body" [2].

As it is less common to stay very close to a mobile phone mast for a long time, working on reducing the phone emission that is carried all day long very close to the human body should have more effect for most users. However, Crainic et al. [3] have investigated parallel cooperative meta-heuristics to reduce exposure to electromagnetic fields generated by mobile phones antennas at planning time whilst still meeting coverage and service quality constraints. It is different than our approach that 
focuses on reducing electromagnetic fields generated by the users' devices at use time.

Algorithms and systems to seamlessly switch between the available networks to remain always best connected are being researched [4-7]. They mainly focus on quality of service (QoS) for decision-making. In this paper, we add another dimension to the network selection issue and underline that electrosmog exposure should also be taken into account.

\section{WIRELESS TECHNOLOGIES SURVEY}

There are different wireless technologies that can be used for communication by the devices carried out by the users. In this section, we survey the main ones including those that are less well-known by the general public but are still important regarding the increased number of wearable communicating sport/health devices products.

\subsection{WI-FI}

The most widespread wireless network technology on portable computer devices is Wi-Fi. Wi-Fi is unlicensed. There are different types of Wi-Fi networks, for example, Wi-Fi IEEE $802.11 \mathrm{~b}$ and $802.11 \mathrm{~g}$ use the $2.4 \mathrm{GHz}$ band and 802.11a uses the $5 \mathrm{GHz}$ band. $5 \mathrm{GHz}$ signals are absorbed more readily by solid objects in their path due to their smaller wavelength and for the same power they propagate less far than $2.4 \mathrm{GHz}$ signals. The average Wi-Fi range is between $30 \mathrm{~m}$ and $100 \mathrm{~m}$. Mobile computer devices that integrate $\mathrm{Wi}-\mathrm{Fi}$ are not made to seamlessly switch between nearby available Wi-Fi networks. There are a number of security issues with Wi-Fi: WEP encryption has been broken for a while; WPA encryption creates separate channels for each user but most public Wi-Fi access points only ask for authentication and does not encrypt afterwards... Although privacy is beyond the scope of the paper, it is important to note that for privacy's sake and the sensitive aspects of some communicated information such as heart rate profile, secure network should be considered. WiMAX is different than Wi-Fi, is more dedicated to long range systems covering kilometers and is rarely integrated in mobile devices for now. The peak power of Wi-Fi $802.11 \mathrm{~b} / \mathrm{g}$ is $100 \mathrm{~mW}$ and 802.11a is $1 \mathrm{~W}$. Kang and Gandhi [8] found that SAR nearfield exposure to a Wi-Fi $100 \mathrm{~mW}$ patch antenna radiated from a laptop computer placed $10 \mathrm{~mm}$ below planar phantom is 2.82 $\mathrm{W} / \mathrm{kg} 1 \mathrm{~g} \mathrm{SAR}$ and $1.61 \mathrm{~W} / \mathrm{kg} 10 \mathrm{~g} \mathrm{SAR}$ at $2.45 \mathrm{GHz}$ and at 5.25 $\mathrm{GHz}$ is $1.64 \mathrm{~W} / \mathrm{kg} 1 \mathrm{~g}$ SAR and $0.53 \mathrm{~W} / \mathrm{kg} 10 \mathrm{~g}$ SAR. A French organization study found that all Wi-Fi $2.4 \mathrm{GHz}$ cards studied are under $2 \mathrm{~W} / \mathrm{kg} 10 \mathrm{~g}$ SAR limit from 0.017 to $0.192 \mathrm{~W} / \mathrm{kg}$ [9] at less than $12.5 \mathrm{~cm}$

\subsection{GSM, UMTS/GPRS, 3G...}

Regarding mobile phones, although more and more smartphones integrate $\mathrm{Wi}-\mathrm{Fi}$, their most widespread wireless network technology remains the one provided by their telecom operator: GSM (around $900 \mathrm{MHz}$ or $1800 \mathrm{MHz}$; maximum distance to cell tower from $1 \mathrm{~km}$ to $20 \mathrm{~km}$ [10]), GPRS, EDGE, UMTS 3G (around $2 \mathrm{GHz}$; from $144 \mathrm{kB} / \mathrm{s}$ in moving vehicles to more than 2 $\mathrm{MB} / \mathrm{s}$ for stationary users [11]; maximum distance to cell tower from $0.5 \mathrm{~km}$ to $5 \mathrm{~km}[10]) \ldots$ The telecom operators have paid licensed to be able to use them. There is different encryption between each user using a cell. Mobile phones switch seamlessly between GSM/3G cells and more and more mobile phones integrate now Wi-Fi. However, only a few phones and telecom providers allow the users to start a phone call with Wi-Fi and switch seamlessly to GSM/3G when the user leaves the Wi-Fi zone. It is also difficult for users to switch to other networks than the ones provided by their telecom provider. From an energy consumption point of view, according to Balasubramanian et al. [12], for $10 \mathrm{kB}$ data size, GSM consumes around 3.25 times less than $3 \mathrm{G}$ and 1.5 times less than $\mathrm{Wi}-\mathrm{Fi}$ (if the cost of scan and transfer is taken into account). However, for $500 \mathrm{kB}+$ data size, GSM consumes as much as $3 \mathrm{G}$ and twice more than Wi-Fi (even if the cost of scan and transfer is taken into account). 3G consumes around 1.9 times more than Wi-Fi (if the cost of scan and transfer is taken into account). It is worth noting that the energy spent for GSM/3G networks can vary a lot depending on the distance between the user and the network antenna as the distance can be quite long compared to $\mathrm{Wi}-\mathrm{Fi}$ : for example for GSM 900, a phone power output may be reduced by 1000 times if it is close to the base station with good signal. The peak handset power limit for GSM 900 is 2 Watts and 1 Watt for GSM 1800. The peak power of $3 \mathrm{G}$ UMTS is $125 \mathrm{~mW}$ at $1.9 \mathrm{GHz}$. It has been found that in rural area, the highest power level for GSM was used about $50 \%$ of the time, while the lowest power was used only $3 \%$ of the time. The corresponding numbers for the city area were approximately $25 \%$ and $22 \%$. The results showed that high mobile phone output power is more frequent in rural areas whereas the other factors (length of call, moving or stationary, indoor or outdoor) were of less importance [13]. Factors that may influence the power control are the distance between hand set and base station and attenuation of the signal, the length of calls (the phone transmits on the maximum allowed power level at the onset of each call), and change of connecting base station, "hand-over', (the phone will temporarily increase output power when connecting to a new base station). Hand-overs will be made when the mobile phone is moved from one cell covered by one base station to another cell, but may also occur on demand from the base station owing to a high load on the network at busy hours. The iPhone $3 \mathrm{G}$ user guide indicates that $10 \mathrm{~g}$ SAR is $0.235 \mathrm{~W} / \mathrm{kg}$ for GSM 900, $0.780 \mathrm{~W} / \mathrm{kg}$ for GSM 1800, 0.878 for UMTS 2100 ; and 0.371 for Wi-Fi. It was worse for the iPhone with $1.388 \mathrm{~W} / \mathrm{kg}$ 1g SAR for UMTS 1900 and $0.779 \mathrm{~W} / \mathrm{kg}$ 1g SAR for Wi-Fi. Combined with the fact that its user guide mentioned that it might be higher at closer than $1.5 \mathrm{~cm}$ and that both $3 \mathrm{G}$ and Wi-Fi may be enabled at the same time, it means that the iPhone can have a $1 \mathrm{~g}$ SAR much higher than the $1.6 \mathrm{~W} / \mathrm{kg}$ limit: above $1.388+$ $0.779=2.167 \mathrm{~W} / \mathrm{kg}$

\subsection{Bluetooth, Zigbee, ANT...}

Bluetooth, based on IEEE 802.15.1, also uses the $2.4 \mathrm{GHz}$ band with a data rate of around $1 \mathrm{MB} / \mathrm{s}$. A large number of mobile phones integrates Bluetooth. Discovery and association of Bluetooth devices are not designed to be seamless. Bluetooth $2.1+$ pairing uses a form of public key cryptography and is less prone to Wi-Fi types of attacks [14]. The peak power of Bluetooth ranges from $1 \mathrm{~mW}$ to $2.5 \mathrm{~mW}$. The normal range of Bluetooth is around $10 \mathrm{~m}$, which is lower than Wi-Fi. With lower distances, Bluetooth has lower consumption than Wi-Fi: around 3 to 5 times more according to Su Min et al. [15]. However, for resource constrained wearable devices such as heart belts and cardio/GPS watches, Bluetooth is still consuming too much energy. It is the reason that a new Bluetooth specification called "Bluetooth low energy" has been released recently and would consume between $1 \%$ to $50 \%$ of normal Bluetooth depending on the application [16]. "Bluetooth low energy" is more seamless: it can support 
connection setup and data transfer as low as $6 \mathrm{~ms}$. "Bluetooth low energy" can use AES-128 encryption. As Bluetooth consumed too much energy for resource constrained devices, other networking technologies have been used. Zigbee based on IEEE 802.15.42003 runs at $868 \mathrm{MHz}$ in Europe, $915 \mathrm{MHz}$ in the USA and Australia, and $2.4 \mathrm{GHz}$ in most other places. Zigbee consumes around 10 to 14 times less than Wi-Fi according to Su Min et al. [15]. The downsize of Zigbee is that it has a much lower data rate from $20 \mathrm{kB} / \mathrm{s}$ to $250 \mathrm{kB} / \mathrm{s}$. Another main network technology that was used in many sport/health monitoring devices is ANT, which is proprietary. ANT and Zigbee can send data in less than 10ms. However, ANT can send bigger files faster as its transmission is 1 $\mathrm{MB} / \mathrm{s}$, which means lower energy to submit large files than Zigbee [17]. Fyfe reports even lower energy consumption for ANT compared to Zigbee for small data size ( 8 bytes) [18]. Anyway, Zigbee and ANT are not available on mobile phones. "Bluetooth low energy" seems a good candidate to replace ANT and Zigbee due to its openness and number of products already using Bluetooth. Martínez-Búrdalo et al. [19] have found that Bluetooth generates very low $10 \mathrm{~g}$ SAR of around $0.037 \mathrm{~W} / \mathrm{kg}$, unfortunately none of these networking technologies are considered as main connecting technologies maybe due to their limited range.

\subsection{Comparison Summary}

Table 1. Networking Technologies Comparison

\begin{tabular}{|c|c|c|c|c|c|c|c|c|}
\hline $\begin{array}{c}\text { Network } \\
\text { Technology }\end{array}$ & 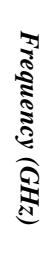 & 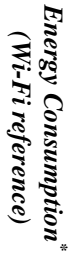 & 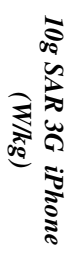 & 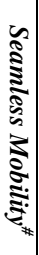 & 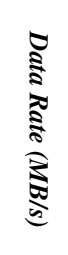 & 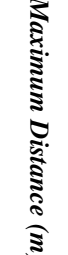 & 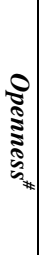 & 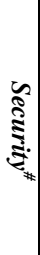 \\
\hline$W i-F i$ & $\begin{array}{c}{[2.4} \\
; 5]\end{array}$ & 1 & 0.371 & $\mathrm{~L}$ & $\begin{array}{l}{[11 ;} \\
54]\end{array}$ & $\begin{array}{l}{[30 ;} \\
150]\end{array}$ & $\mathrm{H}$ & $\mathrm{L}$ \\
\hline$G S M$ & $\begin{array}{c}0.9 \\
; \\
1.8]\end{array}$ & $\begin{array}{c}{[0.67 ;} \\
2]\end{array}$ & $\begin{array}{c}{[0.235 ;} \\
0.78]\end{array}$ & $\mathrm{H}$ & 0.0096 & $\begin{array}{l}{[1000 ;} \\
20000]\end{array}$ & L & M \\
\hline $3 G$ & 2 & $\begin{array}{c}1.8 ; \\
2]\end{array}$ & 0.878 & $\mathrm{H}$ & $\begin{array}{c}{[0.144 ;} \\
2]\end{array}$ & $\begin{array}{l}{[500} \\
5000]\end{array}$ & $\mathrm{L}$ & M \\
\hline Bluetooth & 2.4 & 0.25 & $\sim 0.037$ & $\mathrm{~L}$ & {$[1 ; 3]$} & 10 & $\mathrm{H}$ & M \\
\hline Zigbee & $\begin{array}{c}{[0.8} \\
; \\
2.4]\end{array}$ & 0.085 & $\mathrm{n} / \mathrm{a}$ & M & $\begin{array}{c}{[0.02 ;} \\
0.25]\end{array}$ & 100 & M & M \\
\hline$A N T$ & 2.4 & 0.017 & $\mathrm{n} / \mathrm{a}$ & M & 1 & 30 & $\mathrm{~L}$ & M \\
\hline $\begin{array}{c}\text { Bluetooth } \\
\text { Low Energy }\end{array}$ & 2.4 & $\begin{array}{l}{[0.01} \\
0.125]\end{array}$ & $\mathrm{n} / \mathrm{a}$ & M & 1 & 10 & $\mathrm{H}$ & M \\
\hline
\end{tabular}

*: The energy consumption comparison is roughly derived from the results and information given in the references cited in this paper.

\#: L: Low; M: Medium; H: High / : estimated based on [19]

n/a: non-available

\section{CAREFUL NETWORK SELECTION}

Our goal is to minimise the exposure of the mobile user to electromagnetic radiations while still allowing the users to benefit from the communication of their devices with enough quality of service.
Based on the networking technologies that we have surveyed in the previous sections, the exposure can be significantly reduced by choosing among the different networking technologies available. On recent mobile phones, there are 4 main choices: GSM, 2G, 3G and Wi-Fi. However, it may be cumbersome for the user to learn which networking technology to choose depending on what they are doing with their phone and to constantly manually switch from one network to another. Fortunately, recent mobile phone operating systems such as Android provide an Application Programming Interface (API) that allows third-party applications to switch from one networking technology to another. In this section, we first describe our network selection model and its algorithms and then we explain how we have validated our approach with a proof-of-concept application implemented on an Android phone.

\subsection{Network Switching Selection Model and Algorithms}

We define Ni a network $\mathrm{i}$ among a set of $\mathrm{n}$ available networks in $[1 ; \mathrm{n}]$.

Each network $\mathrm{Ni}$ is associated with a $10 \mathrm{~g}$ SAR in W/kg defined as SARi for the specific device carried out by the user. The related work surveyed above has underlined that different mobile devices have different SARs.

In this case, the optimal policy to minimise the electromagnetic radiation from the mobile device is to select the network with the lower SAR. The algorithm in pseudo-code is:

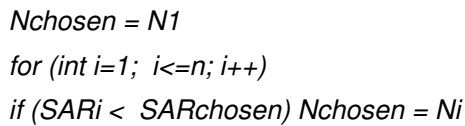

That type of policy works well for voice call activities since the duration time depends on the length of the conversation. However, for other activities that can be carried out faster with faster networking technologies, such data exchange (file download, health data monitoring transmission...), the data rate of the network should be taken into account. We define the data rate of $\mathrm{Ni}$ as $\mathrm{DRi}$ in $\mathrm{MB} / \mathrm{s}$.

In this case of data exchange activities, the optimal policy to minimize electromagnetic radiation is different. The file size of the data to be exchanged is the same for all networks. If we define the time of exposure with $\mathrm{Ni}$ as Ti and FS the file size of the data, we have:

\section{$T i=F S / D R i$}

If we define the exposure during that data exchange with $\mathrm{Ni}$ as $\mathrm{Ei}$, for the optimal policy that would choose $\mathrm{Ni}$, we want $\mathrm{Ei}<=\mathrm{Ej}$ for all $\mathrm{j}$ different than $\mathrm{i}$ in $[1 ; \mathrm{n}]$.

$$
\begin{aligned}
& E i=S A R i{ }^{*} T i \\
& E i<=E j \\
& S A R i{ }^{*} T i<=S A R j{ }^{*} T j \\
& S A R i{ }^{*}(F S / D R i)<=S A R j{ }^{*}(F S / D R j) \\
& S A R i<=(D R i / D R j){ }^{*} S A R j
\end{aligned}
$$

The corresponding pseudo-code is then:

Nchosen $=\mathrm{N} 1$

for (int $i=1 ; i<=n ; i++$ )

if $(S A R i<((D R i / D R c h o s e n) *$ SARchosen $))$ Nchosen $=\mathrm{Ni}$ 
The related work surveyed in the previous sections has underlined that some networking technologies may have much lower SARs than the maximum SAR measured and reported in their specification depending on the context of use. For example, for GSM 900, a phone power output may be reduced by 1000 times if it is close to the base station with good signal. Let us define this attenuation depending on the context and each networking technology as Ai for Ni. We assume that the activity happens in the same context from its start to its end. For example, the user is not moving during the activity, hence the distance between the mobile device and the base station does not change.

In this case, the pseudo-code for "voice call" activity optimal policy becomes:

Nchosen $=\mathrm{N} 1$

for (int $i=1 ; i<=n ; i++$ )

if $(S A R i<(($ Achosen/Ai) * SARchosen $))$ Nchosen = Ni

The pseudo-code for "data exchange" activity optimal policy becomes:

Nchosen $=\mathrm{N} 1$

for (int $i=1 ; i<=n ; i++$ )

if $(S A R i<((D R i / D R c h o s e n)$ * (Achosen/Ai) * SARchosen $))$ Nchosen $=\mathrm{Ni}$

If seamless hand-over between networking technologies was possible, i.e., the activity would not be stopped when the network becomes unavailable and the next network must be used, and the user would move during the activity, our selection algorithm would be again carried out at time of new hand-over.

\subsection{Proof-of-Concept Validation of the Model and its Algorithms}

In order to validate our previous algorithms, we have investigated how to implement them in an Android Google phone application.

As the SARs are different for each phones, our application must be first configured with the SARs phone's values that phones vendors must provide by law (at least in the US and in Europe) with the specification of their phones. It is done manually for the proof-of-concept but it could be automated by fetching that information on remote servers publishing phones SARs values because the phones' model can be programmatically obtained via the Android API and used to fetch the right values.

Then, our application asks the user which activity is going to be carried out: "voice call" or "data exchange". Depending on that next activity, the right policy is chosen, i.e., "voice call" policy or "data exchange" policy. The next user activity may be automatically inferred and when a new activity is detected the networking selection could be automatically triggered but this is beyond the scope of the proof-of-concept prototype.

There are four main networking technologies that can be chosen on current smart phones: 3G, 2G, GSM and Wi-Fi. The Android API facilitates getting information about the different networks available in proximity:

http://developer.android.com/intl/fr/reference/android/net/Connectivi tyManager.html\#getAl/NetworkInfo()

http://developer.android.com/int//fr/reference/android/net/wifi/WifiMa nager.html\#getScanResults()
Few phones and telecom providers allow the users to make phone calls directly through Wi-Fi. So we assume that Wi-Fi is not possible in our proof-of-concept prototype for "voice call" activity. In addition, the Android API does not have an API to force switching to one or another of the available GSM, 2G or 3G networks. So in the "voice call" activity case, we can only display a message to the users explaining that they should manually select a GSM network or if not possible set the "Use Only $2 \mathrm{G}$ Networks" settings.

Concerning the "data exchange" activity, we use the data rate in $\mathrm{MB} / \mathrm{s}$ returned by the following method:

http://developer.android.com/intl/fr/reference/android/net/wifi/Wifilnf o.html\#getLinkSpeed()

As no Android API returns the data rate of GSM, $2 \mathrm{G}$ or $3 \mathrm{G}$ networks, we use a $3 \mathrm{G}$ fixed data rate of $0.5 \mathrm{MB} / \mathrm{s}$ and of 0.0096 $\mathrm{MB} / \mathrm{s}$ for GSM. Future versions of the API may provide more information about the type of network found and we could use finer-grained data rates from this information, for example, it may return a dynamically inferred $2 \mathrm{G}$ data rate.

If the outcome of the algorithm suggests using one of the $\mathrm{Wi}-\mathrm{Fi}$ networks, the Android API facilitates programmatically switching to this Wi-Fi network thanks to the following methods:

http://developer.android.com/int//fr/reference/android/net/wifi/WifiMa nager.html\#enableNetwork(int, boolean)

If the suggestion is to use GSM, $2 \mathrm{G}$ or $3 \mathrm{G}$, as there is no API to switch to these networks, a message can be displayed to the user who can manually select a GSM network, set the "Use Only 2G Networks" settings or select the potential 3G network.

Concerning the attenuation factor depending on the context, we mainly rely on the distance to the network antenna. Wi-Fi does not change its transmitting power and we assume it can transmit as long as it is listed in the Wi-Fi networks discovered by the Android WifiManager. We could refine that assumption by only selecting Wi-Fi networks with a Received Signal Strength Indication (RSSI) above a threshold by using the following method:

http://developer.android.com/intl/fr/reference/android/net/wifi/Wifilnf o.html\#getRssi()

As the distances to the GSM, 2G and $3 \mathrm{G}$ antennas have a significant impact on the "real" SAR, we have used the signal strength returned by the Android API:

http://developer.android.com/reference/android/telephony/Neighbori ngCellInfo.html\#getRssi()

If the signal strength returned is good, which corresponds to the returned value 31 , i.e., $-51 \mathrm{dBm}$ or greater, we use a 0.01 attenuation factor for the network SAR. Another approach may be to use the GPS locations of the user as given by the mobile phone GPS and of the antenna as given by third-parties information. Further work would also be required to fine tune the SAR diminution depending on the distance and the networking technology.

\section{CONCLUSION}

It is still not sure that the average level of electrosmog experienced by the users is harmful. However, as the precautionary principle is to minimize exposure and the major source remains the electromagnetic radiations emitted by the 
user's mobile devices, it is worthwhile trying to minimize their radiations.

With new smartphones, it is possible to switch to one or another of the networking technologies available. We have used this possibility to select the networking technologies depending on their characteristics and the context (user's next activity, distance between the user and the base stations...) in order to minimize these radiations.

There are still a few functionalities to fine-tune and automate but such a network selection switching approach should be considered by the manufacturers of mobile communicating devices worn close to the human body if they want to say that they cared about the precautionary principle.

\section{ACKNOWLEDGMENTS}

This work is sponsored by the European Union, which funds the FP7-ICT-2007-2-224024 PERIMETER project [5].

\section{REFERENCES}

[1] J. Vanderstraeten, "Champs et ondes GSM et santé: revue actualisée de la littérature ". Bruxelles, BELGIQUE: Association des médecins anciens étudiants de l'Université libre de Bruxelles, 2009.

[2] Apple. (2009, accessed on 04/12/2009). Guide d'informations iPhone 3GS. Available:

http://manuals.info.apple.com/fr_FR/iPhone_3GS_informations_i mportantes_F.pdf

[3] T. G. Crainic, B. Di Chiara, M. Nonato and L.,

"Tackling electrosmog in completely configured $3 \mathrm{G}$ networks by parallel cooperative meta-heuristics," Wireless Communications, IEEE, vol. 13, pp. 34-41, 2006.

[4] Q. Song and J. Abbas, "Network selection in an integrated wireless LAN and UMTS environment using mathematical modeling and computing techniques," Wireless Communications, IEEE, vol. 12, pp. 42-48, 2005.

[5] PERIMETER. (accessed on 04/12/2009). Available: http://www.ict-perimeter.eu

[6] H. Liu, C. Maciocco, V. Kesavan and A. L. Y. Low, "IEEE 802.21 Assisted Seamless and Energy Efficient Handovers in Mixed Networks," ed, 2009, pp. 27-42.

[7] M. Kassar, B. Kervella and G. Pujolle, "An overview of vertical hand-over decision strategies in heterogeneous wireless networks," Computer Communications, vol. 31, pp. 2607-2620, 2008.
[8] K. Gang and O. P. Gandhi, "Effect of dielectric properties on the peak 1-and 10-g SAR for $802.11 \mathrm{a} / \mathrm{b} / \mathrm{g}$ frequencies 2.45 and 5.15 to $5.85 \mathrm{GHz}$," Electromagnetic Compatibility, IEEE Transactions on, vol. 46, pp. 268-274, 2004.

[9] Supélec, "Etude « RLAN et Champs électromagnétiques » : synthèse des études conduites par Supélec," 2006

[10] G. Maile, "Impact of UMTS," in Conference on Mobile Networks \& the Environment, 2000.

[11] Wikipedia. (accessed on 04/12/2009). 3G. Available: http://en.wikipedia.org/wiki/3G

[12] N. Balasubramanian, A. Balasubramanian and A.Venkataramani, "Energy consumption in mobile phones: a measurement study and implications for network applications," presented at the Proceedings of the 9th ACM SIGCOMM conference on Internet measurement conference, Chicago, Illinois, USA, 2009.

[13] L. Hillert, A. Ahlbom, D. Neasham, M. Feychting, L. Jarup, R. Navin and P. Elliott, "Call-related factors influencing output power from mobile phones," J Expos Sci Environ Epidemiol, vol. 16, pp. 507-514, 2006.

[14] T. G. Xydis and S. Blake-Wilson, "Security Comparison: BluetoothTM Communications vs. 802.11," ed: Bluetooth Security Experts Group, 2002.

[15] S. M. Kim, J. W. Chong, B. H. Jung, M. S. Kang and D. K. Sung, "Energy-Aware Communication Module Selection through ZigBee Paging for Ubiquitous Wearable Computers with Multiple Radio Interfaces," in Wireless Pervasive Computing 2nd International Symposium, 2007.

[16] Wikipedia. (accessed on 04/12/2009). Bluetooth low energy. Available: http://en.wikipedia.org/wiki/Bluetooth_low_energy

[17] R. Morris. (2008, accessed on 04/12/2009). Comparing ANT and ZigBee. Available:

http://www.embedded.com/products/softwaretools/206900253

[18] K. Fyfe. (2009, accessed on 04/12/2009). Low-power wireless technologies for medical applications. Available: http://acamp.ca/alberta-micro-nano/.../Ken-Fyfe-HealthMedicalDec09.pdf

[19] M. Martínez-Búrdalo, A. Martín, A. Sanchis and R. Villar, "FDTD assessment of human exposure to electromagnetic fields from $\mathrm{WiFi}$ and bluetooth devices in some operating situations," Bioelectromagnetics, vol. 30, pp. 142-151, 2009. 